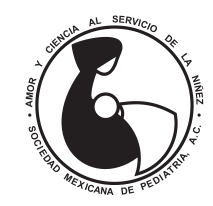

\title{
Hemangioendotelioma kaposiforme gigante con fenómeno de Kasabach-Merritt
}

\author{
Yuliana Montserrat Medina-López, ${ }^{\ddagger} *$ Claudia Ivette Sánchez-Grijalva, ${ }^{\ddagger}$ \\ José Saúl García-López, ${ }^{\ddagger}$ Lucero Beatriz Campos-Félix, ${ }^{\ddagger}$ Ana Beatriz Calderón-Alvarado ${ }^{\ddagger}$ \\ ${ }^{\ddagger}$ Departamento de Pediatría. Hospital General Regional No. 1 del Instituto Mexicano del Seguro Social. Ciudad Obregón, \\ Sonora, México.
}

\begin{abstract}
RESUMEN
Introducción: El hemangioendotelioma kaposiforme es un tumor vascular raro de crecimiento rápido, su principal complicación es el fenómeno de Kasabach-Merritt que se caracteriza por una trombocitopenia grave, coagulopatía por consumo y púrpura. Caso clínico: Se expone el caso de un recién nacido en quien desde el nacimiento se detectó aumento de volumen en extremidad superior izquierda. Mediante resonancia magnética se llegó al diagnóstico. Se inició manejo con esteroides y quimioterapia; sin embargo, la evolución fue tórpida y falleció en la primera semana de vida. El diagnóstico se confirmó en autopsia. Conclusiones: En pacientes con tumoración vascular de crecimiento rápido, se deben buscar alteraciones hematológicas y extensión de la lesión a fin de brindar tratamiento oportuno.
\end{abstract}

Palabras clave: Hemangioendotelioma, Kasabach-Merritt, coagulación intravascular diseminada, recién nacido.

\section{INTRODUCCIÓN}

El hemangioendotelioma es un tumor vascular de presentación rara. No hay incidencia reportada en México; en Estados Unidos de Norteamérica se estima

\footnotetext{
* Correspondencia: YMML, dra.ymedina@gmail.com Conflicto de intereses: Los autores declaran que no tienen. Citar como: Medina-López YM, Sánchez-Grijalva Cl, García-López JS, Campos-Félix LB, Calderón-Alvarado AB. Hemangioendotelioma kaposiforme gigante con fenómeno de Kasabach-Merritt. Rev Mex Pediatr 2020; 87(3):111-114. doi: 10.35366/94842 [Kaposiform haemangioendothelioma with Kasabach-Merritt phenomenon, literature review]
}

\begin{abstract}
Introduction: Kaposiform hemangioendothelioma is a rare, fast growing vascular tumor. Its main complication is the Kasabach-Merritt phenomenon, which is characterized by severe thrombocytopenia, consumption coagulation disorder, and purpura. Case report: We present a male newborn in whom it was detected that the left upper limb was very thickened from birth. Using magnetic resonance imaging, the diagnosis was reached. Management with steroids and chemotherapy was started, however the evolution was torpid and he died in the first week of life. The diagnosis was confirmed at autopsy. Conclusions: Patients with a rapidly growing vascular tumor should look for hematological alterations its extent in order to give timely treatment.
\end{abstract}

Keywords: Haemangioendothelioma, Kasabach-Merritt, disseminated intravascular coagulation, newborn.

una incidencia de 0.07/100,000 nacidos por año. ${ }^{1}$ Este tumor tiende a ser de crecimiento rápido, y cuando se asocia al fenómeno de Kasabach-Merritt, incrementa la mortalidad. ${ }^{2,3}$

Se presenta el caso de un recién nacido $(\mathrm{RN})$ con hemangioendotelioma, dado que es una entidad rara $\mathrm{y}$, que hasta donde sabemos no hay reportes de casos en México.

\section{PRESENTACIÓN DEL CASO}

Recién nacido masculino de dos días de edad, el cual ingresó trasladado de otro hospital por tumoración en brazo izquierdo. Sin antecedentes heredofamiliares de importancia. Producto de primera gesta. Control 
prenatal adecuado, la madre presentó infección de vías urinarias en el último mes, recibió manejo. Ultrasonido prenatal un mes previo al nacimiento reportó extremidad torácica con edema en parte distal, lo cual no se había observado en ultrasonidos anteriores. Nace por vía vaginal, expulsivo prolongado, peso de 3,350 gramos, talla $51 \mathrm{~cm}$, perímetro cefálico $34 \mathrm{~cm}$, perímetro torácico $30 \mathrm{~cm}$, pie $7.5 \mathrm{~cm}$, perímetro brazo izquierdo tercio medio distal $27 \mathrm{~cm}$, Apgar 6-9, Silverman Anderson 0-0, Capurro de 40 semanas. Biometría hemática al nacimiento con plaquetas de 27 mil, por lo que se transfundió concentrado plaquetario. Se decidió envío a nuestro hospital.

A su ingreso se recibió a paciente de dos días de vida, con los siguientes hallazgos: induración violácea circunferencial en brazo izquierdo, la cual genera dolor, con múltiples petequias en antebrazo y mano (Figura 1), además de lesión violácea indurada, redonda en talón derecho, soplo sistólico grado II/VI, el resto de la exploración física sin alteraciones. Se inició manejo por sospecha de sepsis temprana con ampicilina y amikacina por siete días.

Fue valorado al segundo día de vida por ortopedia, quienes solicitaron estudio de imagen simple y contrastada. Hematología pediátrica, por los datos clínicos y por laboratorio sospechan de hemangioendotelioma

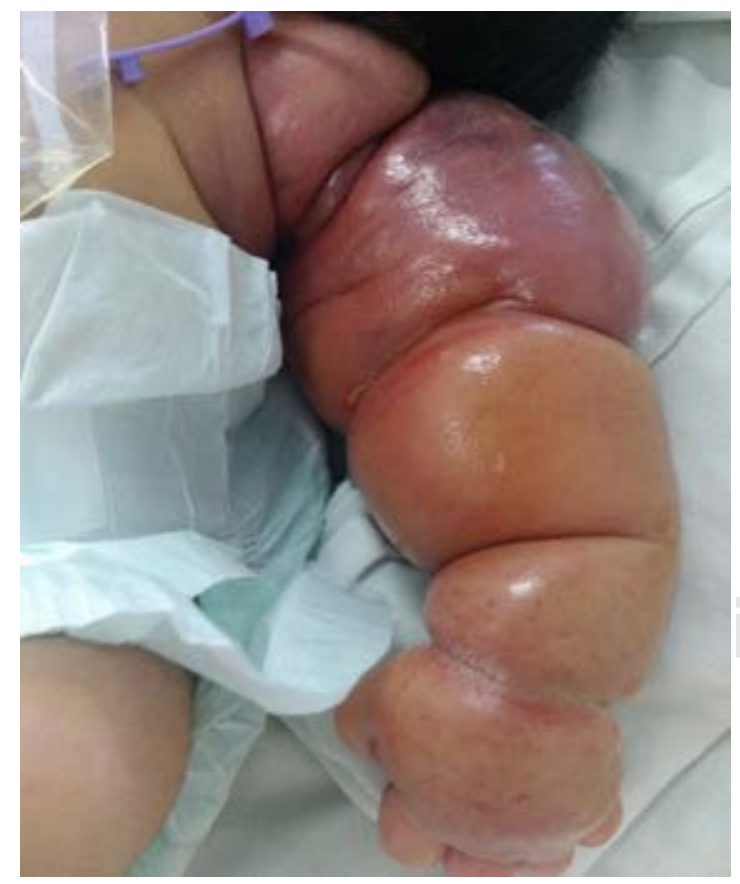

Figura 1: Lesión en extremidad superior izquierda a su ingreso.

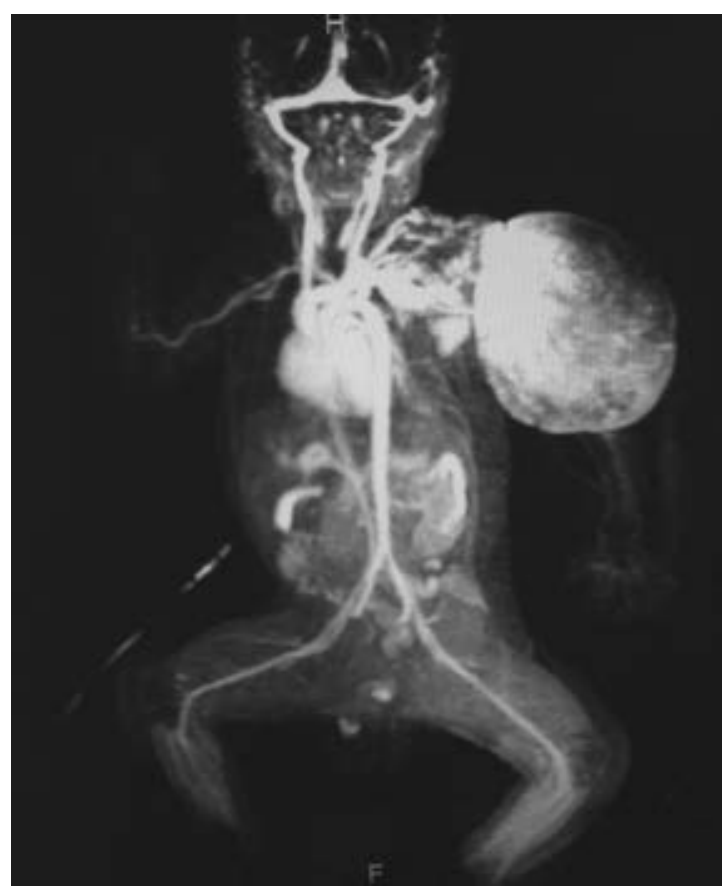

Figura 2: Angiorresonancia con lesión vascular que afecta $80 \%$ de la extremidad superior izquierda, se extiende a tórax pasando la línea media.

kaposiforme con fenómeno de Kasabach-Merritt, solicitando resonancia magnética (IRM). Se pidió valoración a cirugía pediátrica y angiología; sin embargo, no fue candidato para manejo local de resección o embolización por extensión de la lesión.

Se inició manejo con esteroide intravenoso a base de metilprednisolona $2 \mathrm{mg} / \mathrm{kg} /$ día, se suspendieron concentrados plaquetarios y se solicitó panel de coagulación por alto riesgo de coagulación intravascular diseminada. A los tres días de vida, en ultrasonido Doppler de extremidad afectada se observó flujo arterial distal presente, aunque disminuido, y flujo venoso aumentado. En la IRM al cuarto día de vida: lesión vascular que afecta $80 \%$ de extremidad superior izquierda, que se extiende a tórax pasando la línea media (Figura 2).

Por lo anterior, se decidió agregar quimioterapia a base de vincristina $0.05 \mathrm{mg} / \mathrm{kg} /$ día. Sin embargo, el paciente presentó mal estado general; clínicamente con notable incremento de volumen en miembro torácico izquierdo, indurado, tenso, brillante, con coloración violácea de brazo y eritema en antebrazo, dolor a la movilización de la extremidad, sin palparse pulsos en dicho sitio, manteniendo llenado capilar de dos segun- 


\begin{tabular}{|c|c|c|c|c|c|c|c|c|}
\hline Parámetro/fecha & Ingreso & Día 1 & Día 3 & Día 4 & Día 4 & Día 5 & Día 6 & Día 7 \\
\hline Eritrocitos $\left(10^{6} \mu \mathrm{L}\right)$ & 2.83 & 2.75 & 2.93 & 2.38 & 1.94 & 2.87 & 2.97 & 2.66 \\
\hline $\mathrm{Hb}(\mathrm{g} / \mathrm{dL})$ & 9.67 & 9.56 & 10.10 & 8.20 & 6.70 & 9.74 & 10.04 & 9.08 \\
\hline Hto $(\%)$ & 29.80 & 28.70 & 29.60 & 23.80 & 19.30 & 28.40 & 29.30 & 26.30 \\
\hline VCM (fL) & 105.30 & 104.20 & 100.90 & 100.00 & 99.60 & 98.80 & 98.70 & 99.00 \\
\hline $\mathrm{HCM}(\mathrm{pg})$ & 34.20 & 34.80 & 34.30 & 34.60 & 34.60 & 33.90 & 33.80 & 34.20 \\
\hline $\mathrm{CMHC}(\mathrm{g} / \mathrm{dL})$ & 32.40 & 33.40 & 34.00 & 34.60 & 34.70 & 34.30 & 34.20 & 34.50 \\
\hline Leucocitos $\left(10^{3} \mu \mathrm{L}\right)$ & 12.30 & 13.70 & 10.10 & 12.60 & 8.00 & 6.70 & 3.90 & 4.80 \\
\hline Neutrófilos ( $\left.10^{3} \mu \mathrm{L}\right)$ & 6.86 & 6.43 & 6.80 & 5.40 & 4.60 & 3.94 & 2.62 & 3.63 \\
\hline Linfocitos $\left(10^{3} \mu \mathrm{L}\right)$ & 3.76 & 4.85 & 2.10 & 6.00 & 2.20 & 1.86 & 0.96 & 0.82 \\
\hline Plaquetas $\left(10^{3} \mu \mathrm{L}\right)$ & 8.80 & 75.00 & 10.00 & 6.00 & 11.00 & 26.30 & 19.80 & 20.80 \\
\hline VMP (fL) & 14.20 & 9.36 & 10.90 & 8.70 & & 12.23 & 12.99 & 14.05 \\
\hline $\mathrm{TP}$ & & 17.00 & 13.80 & & 15.70 & 18.10 & 15.90 & 16.80 \\
\hline TTPa & & 33.60 & 32.90 & & 37.00 & 32.00 & 31.60 & 32.80 \\
\hline Fibrinógeno & & 221.00 & 164.00 & & 81.00 & 144.00 & 176.00 & 114.00 \\
\hline Dímero D & & 6,446 & 5,983 & & 5,894 & 4,887 & 4,594 & \\
\hline
\end{tabular}

dos, además de petequias en rostro y en miembro torácico derecho. Cursó con datos de hipoperfusión tisular periférica, ingresando a Unidad de Cuidados Intensivos Neonatales (UCIN). Se decidió manejo avanzado de la vía aérea, se inició sedación y analgesia, se cambió esquema de antibióticos, (cefotaxima y vancomicina), apoyo aminérgico.

Al sexto día, cardiología pediátrica hace diagnóstico de hipertensión pulmonar por hiperflujo pulmonar por cortocircuito extracardiaco; foramen oval cortocircuito de izquierda a derecha, con presión sistólica de la arteria pulmonar de $57 \mathrm{mmHg}$ y fracción de eyección del ventrículo izquierdo (FEVI) de 63\%.

Durante su estancia en la UCIN presentó sangrado activo a través de sonda orogástrica y datos compatibles con coagulopatía de consumo (disminución de conteo de plaquetas, tiempos de coagulación prolongados y dímero D elevado) (Tabla 1). Se dio apoyo con transfusión de hemocomponentes; sin embargo, no respondió al manejo, presentando paro cardiorrespiratorio sin respuesta a maniobras de reanimación avanzada.

Se realizó autopsia, con el siguiente reporte: hemangioendotelioma kaposiforme con fenómeno de Kasabach-Merritt, más coagulación intravascular diseminada (Figura 3).

\section{DISCUSIÓN}

El hemangioendotelioma kaposiforme (HEK) es un tumor vascular raro, tiene características comunes al hemangioma capilar y al sarcoma de Kaposi. Anteriormente, este tipo de tumores tenían una variedad de nombres, como "hemangioendotelioma infantil tipo Kaposi", "hemangioma con sarcoma tipo Kaposi" o simplemente "hemangioendotelioma". ${ }^{3}$

La presentación al nacimiento es la más común, siendo rara la descripción en adultos. Usualmente se observa una lesión extensa unifocal que involucra extremidades, tronco, retroperitoneo o región de cabeza y cuello. Histológicamente, se compone de múltiples lóbulos con capilares coalescentes, compuestos principalmente de células endoteliales; se pueden ver microtrombos de plaquetas, hemosiderina y glóbulos rojos extravasados. ${ }^{2,4,5}$

El fenómeno de Kasabach-Merritt (FKM) aparece generalmente asociado a HEK y no a hemangiomas habituales, lo cual incrementa de manera importante la mortalidad. El término FKM incluye una lesión extensa, trombocitopenia severa (menor a $50 \mathrm{mil}$ ), anemia hemolítica microangiopática y coagulopatía por consumo (fibrinógeno < 100). ${ }^{2,4}$ Habitualmente, es más frecuente en hemangiomas mayores a $5 \mathrm{~cm}$, aunque también hay reportes de lesiones más pequeñas. ${ }^{4}$ 


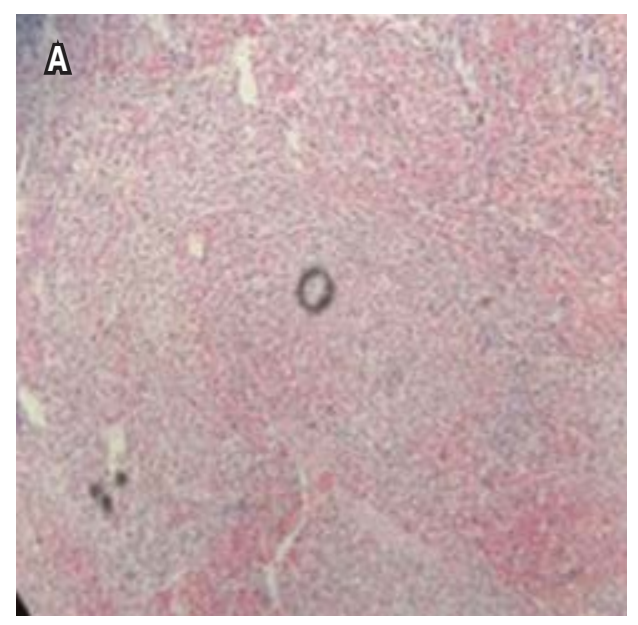

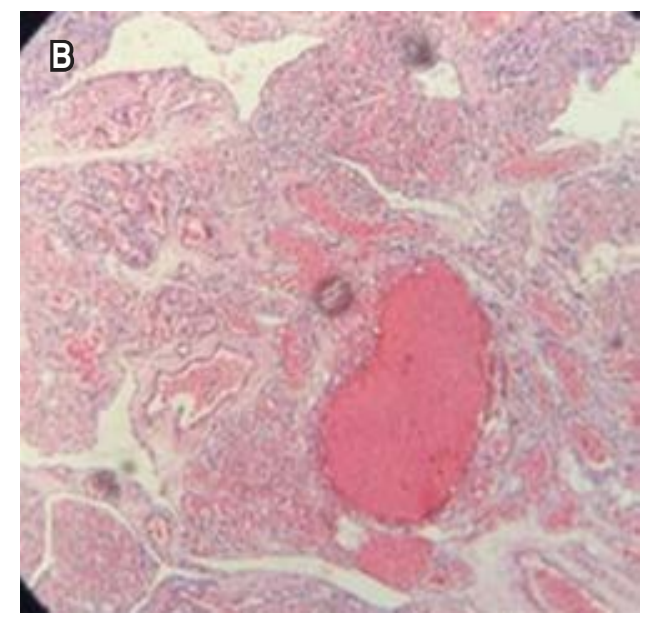

Figura 3:
Autopsia: A)
Hemangioendotelioma
kaposiforme. B) Coagulación
intravascular diseminada.

En HEK complicado por FKM, las lesiones pueden expandirse rápidamente. La piel se vuelve rojo oscuro o púrpura, tensa, con aumento de temperatura y brillante, como sucedió con el paciente. ${ }^{6}$

El diagnóstico se realiza mediante biopsia, sin embargo, cuando ésta se considera peligrosa, se puede realizar el diagnóstico presuntivo basado en estudios de imagen y en la coagulopatía demostrada por laboratorio. ${ }^{4}$

El tratamiento puede ser mediante embolización o compresión mecánica, radioterapia o farmacoterapia; aunque la respuesta al manejo es variable y va de días a semanas. ${ }^{4,6}$ En este caso, el paciente no fue candidato a manejo quirúrgico, ya que la lesión se extendía sobre la línea media, por lo que se decidió iniciar con esteroide y quimioterapia, el cual ha mostrado, según la literatura, respuesta a partir de las cuatro semanas de su inicio. ${ }^{2,4}$ No obstante, por la extensión de la lesión, así como las complicaciones asociadas, el paciente presentó una evolución tórpida y falleció.

\section{REFERENCIAS}

1. Croteau SE, Liang MG, Kozakewich HP, Alomari AI, Fishman SJ, Mulliken JB et al. Kaposiform hemangioendothelioma: atypical features and risk of a Kasabach-Merritt phenomenon in 107 referrals. J Pediatr. 2013; 162(1): 142-147.

2. O'Rafferty C, O'Regan G, Irvine AD, Smith OP. Recent advances in the pathobiology and management of Kasabach-Merritt phenomenon. Br J Haematol. 2015; 171(1): 38-51.

3. Cotran RS, Kumar V, Robbins S. Robbins Patología Estructural y Funcional. 6a ed. Madrid: McGraw-Hill Inc./Interamericana de España; 2005.

4. Adams DM, Brandão LR, Peterman CM, Gupta A, Patel M, Fishman $S$ et al. Vascular anomaly cases for the pediatric hematologist oncologists-An interdisciplinary review. Pediatr Blood Cancer. 2018; 65(1).

5. Arabi, J, López F. Hemangiomatosis neonatal difusa. Presentación de un caso y revisión de la literatura. Rev Mex Pediatr. 2000; 67(6): 270-273.

6. Rodríguez N, Benavides P. Sirolimus (rapamicina) en pacientes con hemangioendotelioma kaposiforme. Caso clínico. Rev Chil Pediatr. 2013; 84(5): 537-544. 